\title{
Interferometry of class I methanol masers, statistics and the distance scale
}

\author{
Maxim A. Voronkov ${ }^{1,3}$, Shari L. Breen ${ }^{2,3}$, Simon P. Ellingsen $^{3}$ and \\ Christopher H. Jordan ${ }^{4}$ \\ ${ }^{1}$ CSIRO Astronomy and Space Science, PO Box 76, Epping, NSW 1710, Australia \\ email: maxim.voronkov@csiro.au \\ ${ }^{2}$ Sydney Institute for Astronomy (SIfA), School of Physics, University of Sydney, Sydney, \\ NSW 2006, Australia \\ ${ }^{3}$ School of Mathematics and Physics, University of Tasmania, GPO Box 252-37, Hobart, Tas \\ 7000, Australia \\ ${ }^{4}$ International Centre for Radio Astronomy Research, Curtin University, Bentley, WA 6845, \\ Australia
}

\begin{abstract}
The Australia Telescope Compact Array (ATCA) participated in a number of survey programs to search for and image common class I methanol masers (at 36 and $44 \mathrm{GHz}$ ) with high angular resolution. In this paper, we discuss spatial and velocity distributions revealed by these surveys. In particular, the number of maser regions is found to fall off exponentially with the linear distance from the associated young stellar object traced by the 6.7-GHz maser, and the scale of this distribution is $263 \pm 15$ milliparsec. Although this relationship still needs to be understood in the context of the broader field, it can be utilised to estimate the distance using methanol masers only. This new technique has been analysed to understand its limitations and future potential. It turned out, it can be very successful to resolve the ambiguity in kinematic distances, but, in the current form, is much less accurate (than the kinematic method) if used on its own.
\end{abstract}

Keywords. masers - ISM: molecules - stars: formation

\section{Introduction}

Early studies of methanol masers empirically divided them into two classes (Batrla et al. 1987): class I are typically scattered around the presumed location of the young stellar object (YSO) over an area often compared with the primary beam of the 20-m class radio telescope, while class II are compact at arcsecond resolution and pinpoint the location of the YSO. These differences were traced to collisional and radiative pumping, respectively (see Voronkov et al. (2014) and references therein for further information). The Australia Telescope Compact Array (ATCA) participated in a number of survey programs, both targeted and blind, to search for and image common class I methanol masers (at 36 and $44 \mathrm{GHz}$ ) with high angular resolution (Voronkov et al. 2014; Jordan et al. 2015, 2017; and unpublished data 36-GHz data from the Methanol Multibeam (MMB) follow-up project). This resulted in a large sample of class I methanol masers studied at sub-arcsecond resolution, and provided us the basis for statistical analysis.

\section{Velocity distribution}

Due to complexity of spatial and kinematic structure of class I methanol masers, we decomposed emission into groups of Gaussian components coincident within $3 \sigma$ in position 
and velocity and used those groups for statistical analysis instead of individual components (see Voronkov et al. (2014) for further details). Each component group was assigned an association with a $6.7-\mathrm{GHz}$ maser (from the MMB project, see Green et al. (2017) and references therein), if found within an arcminute (if there is more than one $6.7-\mathrm{GHz}$ maser in the vicinity we used the nearest). The middle of the velocity range spanned by the $6.7-\mathrm{GHz}$ maser emission is often used as a reference velocity for the kinematic distance estimates. The comparison between this mid-range velocity and velocity of the associated class I emission revealed that the difference is largely (ignoring a few highvelocity components) a Gaussian variate with the standard deviation of $3.65 \pm 0.05$ and $3.32 \pm 0.07 \mathrm{~km} \mathrm{~s}^{-1}$ for the 36 and $44-\mathrm{GHz}$ masers, respectively (Voronkov et al. 2014). The difference between the two standard deviations, if real, may be a consequence of preferential orientation with respect to the line of sight direction for the 36 and 44-GHz masers which belong to two different transition series (Sobolev et al. 2007; see also the paper in this volume). The distribution of velocity offsets has also a small but significant mean of $-0.57 \mathrm{~km} \mathrm{~s}^{-1}$ (class I masers are blue-shifted; uncertainties are 0.06 and $0.07 \mathrm{~km} \mathrm{~s}^{-1}$ for the 36 and 44-GHz masers, respectively). However, the velocities of class I masers show a better agreement with that of the thermal gas: the standard deviation of the velocity offsets with respect to CS is $1.5 \pm 0.1 \mathrm{~km} \mathrm{~s}^{-1}$ with insignificant mean offset (see Jordan et al. 2015,2017 ). Therefore, the velocity of class I methanol masers seems to be a better tracer of the quiescent gas velocity than the velocity derived from the 6.7-GHz spectra. This conclusion is in agreement with the earlier results of Green \& McClure-Griffiths (2011) on the velocity distribution of $6.7-\mathrm{GHz}$ masers.

\section{Spatial distribution}

Despite general scatter around the presumed YSO location, the smaller spatial separations of class I maser emission are somewhat preferred with the region of influence of a YSO being less than 1 pc (see Fig. 29 of Kurtz et al. 2004). The large statistics and availability of homogenous $6.7-\mathrm{GHz}$ data through the MMB survey allowed us to probe this distribution in detail. It turned out that the linear separations between class I maser components and associated 6.7-GHz masers have an exponential distribution with good accuracy (the scale is $263 \pm 15$ milliparsec; see Voronkov et al. 2014). This relationship still needs to be understood in the context of the broader field.

\section{New method to estimate distances}

The empirical distribution of linear separations discussed in the previous section can, in principle, be turned into a statistical distance estimator from the measured angular separations. This method does not use velocities and, therefore, is applicable in the Galactic Centre direction where the kinematic method fails. Here we analyse the limitations of this new approach. First, the number of component groups for each individual source is rarely large. Therefore, it may be more practical to assume that the measured angular separation is the exponential variate for each individual source. Then, one can use the fact that, for the exponential distribution, the mean is an unbiased maximum likelyhood estimator of the scale parameter. And equating the measured mean angular separation $\left(\left\langle d_{\text {arcsec }}\right\rangle\right)$ to the expected linear scale of $263 \pm 15$ milliparsecs (Voronkov et al. 2014 ), one gets the following recipe for the distance $D_{k p c} \approx 54 /\left\langle d_{\text {arcsec }}\right\rangle$. The numbers may be too low for a proper statistical test that the measured angular separations for a given source are indeed consistent with the exponential distribution. However, one could compute the standard deviation and compare it with the mean as a cross-check: they are 


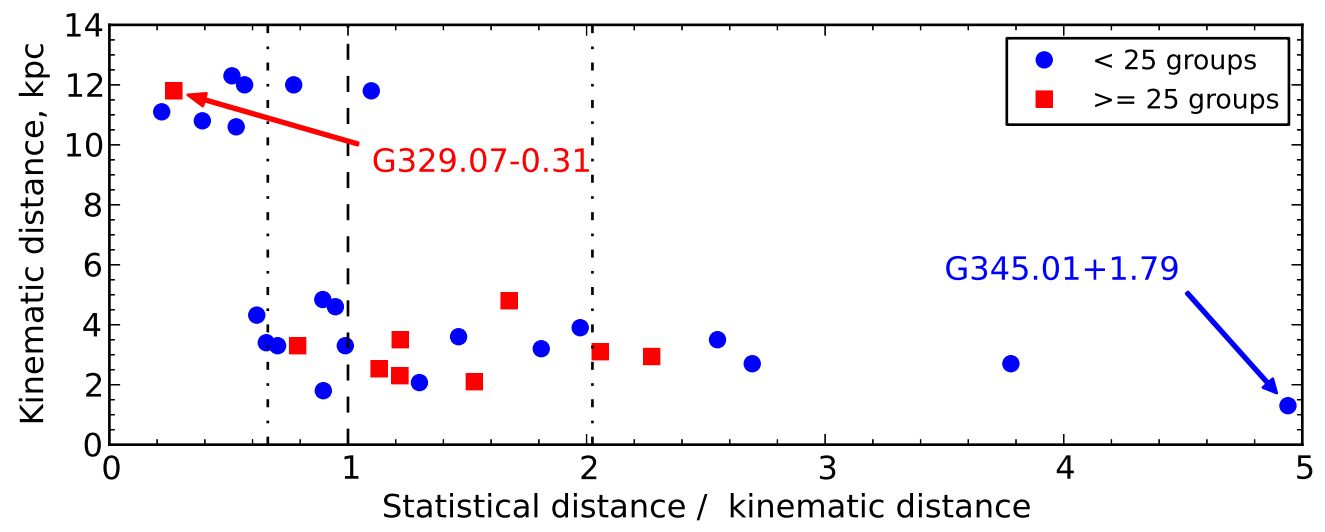

Figure 1. Comparison of the distance estimate based on the empirical spatial distribution of class I masers and the kinematic distance estimate based on the $6.7-\mathrm{GHz}$ velocities. Each point represents a single $6.7-\mathrm{GHz}$ maser from the MMB catalogue associated with at least 15 class I methanol maser component groups (sources with at least 25 such groups are shown by (red) squares). The vertical dash-dotted lines enclose the area where both methods agree (dashed vertical line represents the exact agreement) with the $95 \%$ confidence, under assumption that the new method is the only source of uncertainty and the class I methanol maser has 15 component groups.

identical for the exponential distribution. Note, the uncertainty of the expected linear scale quoted above has rather small contribution (about 5\%) to the total error budget which is dominated by the uncertainty of $\left\langle d_{\text {arcsec }}\right\rangle$ in the case of small number statistics and, quite likely, by the systematics discussed below.

Second, the distribution of linear separations, which this method is based upon, has been obtained for an ensamble of sources. Applying it to individual sources introduces additional systematics due to possible hidden parameters and idiosyncrasies of the sources. In paricular, it is discussed by Voronkov et al. (2014) that more evolved sources show some tendency to be more spread out both spatially and in velocity. This evolutionary trend has been completely ignored in the analysis above. In addition, the ATCA followup of the 44-GHz masers found in the MALT45 blind survey (Jordan et al. 2017) and the new (unpublished) 36-GHz ATCA survey targeting MMB 6.7-GHz masers, both revealed many simple sources. This is an additional evidence suggesting that the source sample studied by Voronkov et al. (2014) may not be representative of the whole population of class I methanol masers (or, even, the subset of such masers located in high-mass star forming regions). Another important systematic factor is the association of class I methanol masers with a range of phenomena producing shocked gas, not just outflows (see discussion in Voronkov et al. 2010; 2014). The spatial distribution of class I masers conceivably depends on the exact mechanism, but details, or even the relative occurrence of different scenarios, are not clear at present.

To examine the accuracy of such statistical distances in practical terms, we compared them with kinematic distances based on the middle of the velocity range of the associated 6.7-GHz maser for the whole sample of Voronkov et al. (2014) where an association between class I and class II masers can be made and where the class I maser emission was decomposed into at least 15 component groups. Each point in Fig. 1 represents a single source. Statistical distances are expected to be more accurate for sources with large number of component groups which are shown as (red) squares. But, in general, points are expected to be confined between two dash-dotted lines on the diagram (agreement 
with the kinematic method with $95 \%$ confidence), or roughly a factor of 2 uncertainty in distances. However, it is evident from Fig. 1 that there are many outliers with grossly overestimated distances for nearby sources and underestimated for sources beyond $10 \mathrm{kpc}$. Inspection of individual sources in the former category reveals two main reasons for overestimated distances: the sensitivity cutoff due to primary beam (e.g. the most extreme outlier point corresponding to G345.01+1.79 which was found by Voronkov et al. (2014) to have emission beyond the half-power point of the primary beam) and questionable associations of the class I maser emission with the $6.7-\mathrm{GHz}$ masers in complex sources. In both cases, the apparent spread of class I maser emission is underestimated causing the method to overestimate the distance. However, it is worth noting that there are many relatively compact sources which have less than 15 component groups. There seems to be a sysematic tendency to overestimate the distances to such sources, although some of them may indeed have been incorrectly assigned to the near kinematic distance. For distant sources, extreme outliers (which this method tries to bring closer) are likely to be largely genuine cases of sources wrongly assigned to the far kinematic distance (see, for example, the discussion on G329.07-0.31 in Voronkov et al. 2014). Therefore, despite having a relatively low accuracy on its own, this method can be used to assist resolving the kinematic distance ambiguity. It can also provide an extra constraint for the Bayesian approach suggested by Reid et al. (2016; see also the paper in this volume).

It is worth noting that this method can be extended to sources without a $6.7-\mathrm{GHz}$ maser or other way to pinpoint the YSO location. The averaged position of all class I maser emission is close to the location of the $6.7-\mathrm{GHz}$ maser for the majority of the sources in our sample (although there are several sources with pronounced asymmetry

for which it is not the case) and can be used as a proxy. This is an additional source of systematic uncertainty, but may be acceptable as the statistical distances are the rough estimates anyway.

\section{References}

Batrla, W., Matthews, H.E., Menten, K.M., \& Walmsley, C.M. 1987, Nature, 326, 49

Green, J.A., Breen, S.L., Fuller, G.A., McClure-Griffiths, N.M., Ellingsen, S.P., Voronkov, M.A. Avison, A., Brooks, K., Burton, M.G., Chrysostomou, A., Cox, J., Diamond, P.J., Gray, M.D., Hoare, M.G., Masheder, M.R.W., Pestalozzi, M., Phillips, C., Quinn, L.J., Richards, A.M.S., Thompson, M.A., Walsh, A.J., Ward-Thompson, D., Wong-McSweeney, D., \& Yates, J.A. 2017, MNRAS, 469, 1383

Green, J.A., \& McClure-Griffiths, N.M. 2011, MNRAS, 368, 1843

Jordan, C.H., Walsh, A.J., Breen, S.L., Ellingsen, S.P., Voronkov, M.A., \& Hyland, L.J. 2017, MNRAS, 471, 3915

Jordan, C.H., Walsh, A.J., Lowe, V., Voronkov, M.A., Ellingsen, S.P., Breen, S.L., Purcell, C.R., Barnes, P., Burton, M.G., Cunningham, M.R., Hill, T., Jackson, J.M., Longmore, S.N., Peretto, N., \& Urquhart, J.S. 2015, MNRAS, 448, 2344

Kurtz, S., Hofner, P., \& Álvarez, C.V. 2004, ApJS, 155, 149

Reid, M.J., Dame, T.M., Menten, K.M., \& Brunthaler, A. 2016, ApJ, 823, 77

Sobolev, A.M., Cragg, D.M., Ellingsen, S.P., Gaylard, M.J., Goedhart, S., Henkel, C., Kirsanova, M.S., Ostrovskii, A.B., Pankratova, N.V., Shelemei, O.V., van der Walt, D.J., Vasyunina, T.S., \& Voronkov, M.A. 2007, in: J.M. Chapman \& W.A. Baan (eds.), Proc. IAU Symp. 242, Astrophysical Masers and their Environments (Cambridge Univ. Press), p. 81

Voronkov, M.A., Caswell, J.L., Ellingsen, S.P., Green, J.A., \& Breen, S.L. 2014, MNRAS, 439, 2584

Voronkov, M.A., Caswell, J.L., Ellingsen, S.P., \& Sobolev, A.M. 2010, MNRAS, 405, 2471 